\title{
Measuring the Spin-Polarizabilities of the Proton in Polarized Compton Scattering at MAMI-Mainz
}

\author{
R. Miskimen \\ Department of Physics \\ University of Massachusetts \\ Amherst, MA 01003 USA \\ E-mail: miskimen@physics.umass.edu
}

Unpolarized Compton scattering data have been used with great success to measure the electric and magnetic polarizabilities of the proton. However relatively little is known about the spin-polarizabilites of the proton, which parameterize how a polarized photon interacts with the spin of the proton. At $\mathrm{O}\left(\omega^{3}\right)$ four spin-polarizabilities $\gamma_{\mathrm{E} 1 \mathrm{E} 1}, \gamma_{\mathrm{M} 1 \mathrm{M} 1}, \gamma_{\mathrm{E} 1 \mathrm{M} 2}$, and $\gamma_{\mathrm{M} 1 \mathrm{E} 2}$ enter the Compton scattering amplitude. The most model independent way to determine the spin-polarizabilities is by measuring Compton scattering asymmetries in the $\Delta$ region with circularly polarized photons and a transverse or longitudinally polarized target, $\Sigma_{2 \mathrm{x}}$ and $\Sigma_{2 \mathrm{z}}$, and by measuring the in-plane/transverse-plane Compton scattering asymmetry on an unpolarized target with linearly polarized photons, $\Sigma_{3}$. This talk will present new Compton scattering data for $\Sigma_{2 \mathrm{x}}$ taken by the A2 Collaboration using the Crystal Ball at Mainz. The incident photon energies ranged from $270 \mathrm{MeV}$ to $2 \pi$ threshold, and the Compton scattering angles ranged from $50^{\circ}$ to $150^{\circ}$. The data are compared with the results of a dispersion theory calculation for Compton scattering, and the sensitivity of the data to the proton spin-polarizabilties are presented.

The 7th International Workshop on Chiral Dynamics

August 6 -10, 2012

Jefferson Lab, Newport News, Virginia, USA 


\section{Introduction}

Polarizabilities tell us about the response of a system when an external electromagnetic field is applied. At $\mathrm{O}\left(\omega^{3}\right)$ nucleon spin-flip operators enter the Compton scattering amplitude, and the constants that multiply the operators are the spin-polarizabilities, given by $\gamma_{\mathrm{E} 1 \mathrm{E} 1}, \gamma_{\mathrm{M} 1 \mathrm{M} 1}, \gamma_{\mathrm{E} 1 \mathrm{M} 2}$, and $\gamma_{\mathrm{MIE2}}$, where the multipoles refer to the incident and outgoing photons.

$$
H_{\text {eff }}^{(3)}=-\frac{1}{2} 4 \pi\left[\gamma_{E 1 E 1} \vec{\sigma} \cdot\left(\vec{E} \times \frac{d}{d t} \vec{E}\right)+\gamma_{M 1 M 1} \vec{\sigma} \cdot\left(\vec{H} \times \frac{d}{d t} \vec{H}\right)-2 \gamma_{M 1 E 2} E_{i j} \sigma_{i} H_{j}+2 \gamma_{E 1 M 2} H_{i j} \sigma_{i} E_{j}\right]
$$

In qualitative terms spin polarizabilities tell us about the response of the nucleon spin to the photon polarization. The "stiffness" of the spin can be thought of as arising from the nucleons spin interacting with the pion cloud. Polarizabilities can be calculated in dispersion theory, in effective field theories to order $\mathrm{O}\left(\omega^{4}\right)$, and in the near future, from lattice calculations. Precision measurements of nucleon polarizabilities are essential in order to extend our knowledge of hadron structure and low-energy QCD.

\section{Existing measurements and theoretical predictions}

Measurements have been made of two combinations of the spin-polarizabilities. The forward spin-polarizability, $\gamma_{0}$, defined in the multipole basis as

$$
\gamma_{0}=-\gamma_{E 1 E 1}-\gamma_{E 1 M 2}-\gamma_{M 1 E 2}-\gamma_{M 1 M 1}
$$

has been evaluated using the Gell-Mann, Goldberger, and Thirring sum rule

$$
\gamma_{0}=\frac{1}{4 \pi^{2}} \int_{m_{\pi}}^{\infty} \frac{\sigma_{3 / 2}-\sigma_{1 / 2}}{\omega^{3}} \mathrm{~d} \omega=(-1.01 \pm .08 \pm .10) \times 10^{-4} \mathrm{fm}^{4}
$$

where the errors are statistical and systematic. The backward spin-polarizabilty, $\gamma_{\pi}$, defined in the multipole basis as

$$
\gamma_{\pi}=-\gamma_{E 1 E 1}-\gamma_{E 1 M 2}+\gamma_{M 1 E 2}+\gamma_{M 1 M 1}=(-8.0 \pm 1.8) \times 10^{-4} \mathrm{fm}^{4}
$$

has been obtained from a dispersive analysis of unpolarized Compton scattering.

Table 1 presents the results from theory and experiment for the spin-polarizabilities. At present, no data exist for the separated spin-polarizabilities in the multipole basis.

\section{Sensitivity of the spin-polarizabilities to Compton scattering asymmetries}

The most model independent determination of the proton spin-polarizabilities is through Compton scattering asymmetries in the $\Delta$ region. Because the spin-polarizability effect goes at $\mathrm{O}\left(\omega^{3}\right)$, energies above pion threshold are needed to amplify the effect. Asymmetries can be measured with incident circularly polarized photons and a transverse or longitudinal polarized target $\left(\Sigma_{2 \mathrm{x}}, \Sigma_{2 \mathrm{z}}\right)$, and through an asymmetry for Compton scattering parallel/perpendicular to the direction of linear polarization $\left(\Sigma_{3}\right)$. Fig. 1 shows a sensitivity plot of the $\Sigma_{2 x}$ asymmetry (transverse polarized target) when the spin polarizabilities are allowed to vary. Cross sections are calculated using the dispersion theory calculation of Drechsel et al. ${ }^{1}$ In the plot on the left, 
$\gamma_{0}$ and $\gamma_{\pi}$ are held fixed at their experimental values, $\gamma_{\mathrm{EIE} 1}$ is held at the nominal value from Ref. [2] and $\gamma_{\mathrm{M} 1 \mathrm{M} 1}$ is allowed to vary from 1.9 to 3.9 in the standard units. For the plot on the right, the same conditions hold, however in this case $\gamma_{\mathrm{M} 1 \mathrm{M} 1}$ is held at the nominal value from Ref. [2] and $\gamma_{\mathrm{E} 1 \mathrm{E} 1}$ is allowed to vary from -3.3 to -5.3 in the standard units. The width of the bands shows the propagated errors from the uncertainties in the $\alpha, \beta, \gamma_{0}$ and $\gamma_{\pi}$. Fig. 1 shows that under conditions where $\alpha, \beta, \gamma_{0}$ and $\gamma_{\pi}$ are constrained by their experimental values, the $\Sigma_{2 \mathrm{x}}$ asymmetry has weak sensitivity to $\gamma_{\mathrm{M} 1 \mathrm{M} 1}$, and good sensitivity to $\gamma_{\mathrm{E} 1 \mathrm{E} 1}$.

\section{Measuring Compton scattering asymmetries in the $\Delta$ region at MAMI-Mainz}

Data were taken using the Crystal Ball and TAPS detectors at Mainz. Fig. 2 shows a schematic view of the detector system. The CB/TAPS system subtends approximately $80 \%$ of $4 \pi$ solid angle, and both the Compton scattered photon and recoil proton are detected. The target was a frozen-spin butanol target, with polarization $\sim 90 \%$ and relaxation time $\sim 1000$ hours. Details of the experimental setup, data taking, and analysis are given in the Ph.D. thesis of P. Martel ${ }^{3}$. Fig. 3 shows a missing mass spectrum from Martel's analysis where the proton mass peak is clearly visible, as are background events at higher missing mass.

Preliminary experimental asymmetries are presented in Fig. 4 for $\Sigma_{2 \mathrm{x}}$. As demonstrated in Fig. 1 , the $\Sigma_{2 \mathrm{x}}$ asymmetry has good sensitivity to the $\gamma_{\mathrm{EIE1}}$ spin-polarizability and small sensitivity to $\gamma_{\mathrm{M} 1 \mathrm{M} 1}$, provided that $\alpha, \beta, \gamma_{0}$ and $\gamma_{\pi}$ are constrained by their experimental values. Based on the position of the asymmetry points on the plot, the statistical errors, and the negligible sensitivity to $\gamma_{\mathrm{M} 1 \mathrm{M} 1}$, we can make a preliminary estimate for $\gamma_{\mathrm{E} 1 \mathrm{E} 1} \approx(-4.5 \pm 1) \times 10^{-4} \mathrm{fm}^{4}$. This value for $\gamma_{\mathrm{EIE} 1}$ is in good agreement with dispersion theory calculations.

\section{Summary and outlook}

The data presented here are the first Compton scattering double-polarization observables on the proton ever measured. The data are sensitive to the $\gamma_{\mathrm{EIE} 1}$ spin-polarizability, and can be used to make a preliminary estimate $\gamma_{\mathrm{EIEl}} \approx(-4.5 \pm 1) \times 10^{-4} \mathrm{fm}^{4}$. This estimate will be revised and presented in a future publication, where the effects of finite energy binning and theoretical model dependence will be taken into account.

One of the objectives of the Compton scattering program at Mainz is to measure the four separated spin-polarizabilities of the proton with an accuracy of approximately $\pm 0.5 \times 10^{-4} \mathrm{fm}^{4}$ through measurements of $\Sigma_{2 \mathrm{x}}, \Sigma_{2 z}$, and $\Sigma_{3}$. In these analyses it will be reasonable to use the experimental constraint on $\gamma_{0}$, because $\gamma_{0}$ is known very well, but not the experimental constraint on $\gamma_{\pi}$, which is known relatively poorly.

Recently, in late 2012, new Compton scattering data were taken at Mainz with linearly polarized photons and a liquid hydrogen target to measure the $\Sigma_{3}$ asymmetry in the $\Delta$ region. Looking towards the future, the frozen-spin target will be reinstalled with longitudinal polarization in summer and fall 2013 and running on $\Sigma_{2 z}$ will commence sometime after that. Over the next 
few years we can expect that polarized Compton scattering measurements at Mainz will allow for the determination of all four proton spin-polarizabilities with reasonable accuracy.

\begin{tabular}{|c|c|c|c|c|c|c|c|c|c|c|c|}
\hline & \multicolumn{3}{|c|}{$\begin{array}{c}\text { Chiral perturbation } \\
\text { theory }\end{array}$} & \multicolumn{2}{c|}{ Lorentz } & \multicolumn{4}{c|}{ Dispersion theory } & \\
\hline & $\mathbf{O}\left(\mathbf{p}^{3}\right)$ & $\mathbf{O}\left(\mathbf{p}^{4}\right)$ & $\mathbf{O}\left(\mathbf{p}^{4}\right)$ & LC3 & LC4 & SSE & BGLMN & HDPV & KS & DPV & Experiment \\
\hline$\gamma_{\text {E1E1 }}$ & -5.7 & -1.4 & -1.8 & -3.2 & -2.8 & -5.7 & -3.4 & -4.3 & -5.0 & -4.3 & - \\
\hline$\gamma_{\text {M1M1 }}$ & -1.1 & 3.3 & 2.9 & -1.4 & -3.1 & 3.1 & 2.7 & 2.9 & 3.4 & 2.9 & - \\
\hline$\gamma_{\text {E1M2 }}$ & 1.1 & 0.2 & .7 & .7 & .8 & .98 & 0.3 & -0.01 & -1.8 & 0 & - \\
\hline$\gamma_{\text {M1E2 }}$ & 1.1 & 1.8 & 1.8 & .7 & .3 & .98 & 1.9 & 2.1 & 1.1 & 2.1 & - \\
\hline$\gamma_{\mathbf{0}}$ & 4.6 & -3.9 & -3.6 & 3.1 & 4.8 & .64 & -1.5 & -.7 & 2.3 & -.7 & $-1.01 \pm 0.08$ \\
\hline$\gamma_{\boldsymbol{\pi}}$ & 4.6 & 6.3 & 5.8 & 1.8 & -.8 & 8.8 & 7.7 & 9.3 & 11.3 & 9.3 & $8.0 \pm 1.8$ \\
\hline
\end{tabular}

Table 1. Theory and experiment for the proton spin-polarizabilities. Units are $10^{-4} \mathrm{fm}^{4} . \mathrm{O}\left(\mathrm{p}^{\mathrm{n}}\right)$ are CPT calculations [4][5][6]. LC3 and LC4 are $\mathrm{O}\left(\mathrm{p}^{3}\right)$ and $\mathrm{O}\left(\mathrm{p}^{4}\right)$ Lorentz invariant CPT calculations, respectively [7]. SSE is a calculation in the small-scale-expansion [4]. BGLMN, HDPV, KS, and DPV are dispersion relation calculations [8][2][9][1]. The data for $\gamma_{0}$ and $\gamma_{\pi}$ are from [10] and [11], respectively.

\section{References}

[1] D. Drechsel, B. Pasquini, M. Vanderhaeghen, Phys. Rep. 378, 015204 (2003).

[2] B. Holstein, D. Drechsel, B. Pasquini, M. Vanderhaeghen, Phys. Rev. C 61, 034316 (2000).

[3] P. Martel, $\mathrm{PhD}$ thesis, University of Massachusetts, 2012.

[4] T.R. Hemmert, B.R. Holstein, J. Kambor, and G. Knochlein, Phys. Rev. D 57, 5746 (1998)

[5] V. Vijaya Kumar, J. McGovern, M. Birse, Phys. Lett B 479, 167 (2000).

[6] G. Gellas, T. Hemmert, Ulf-G. Meißner, Phys. Rev. Lett 86, 14 (2001)

[7] D. Djukanovic, PhD thesis, Johannes Gutenberg Universitat Mainz, Germany, June 2008.

[8] D. Babusci, G. Giordano, A. L’vov, G. Matone, A. Hathan, Phys. Rev. C 58, 1003 (1998).

[9] S. Kondratyuk, O. Scholten, Phys. Rev. C 64, 024005 (2001).

[10]GDH and A2 collaborations, Phys. Rev. Lett. 87,2 (2001); H. Dutz, et al., Phys. Rev. Lett 91, 19 (2003). 
[11] M. Camen et al., Phys. Rev. C 65, 0302202 (2002); M. Schumacher, Prog. Part. Nucl. Phys. 55, 567 (2005); B. Pasquini, D. Drechsel, M. Vanderhaeghen, Phys. Rev. C 76, 015203 (2007).
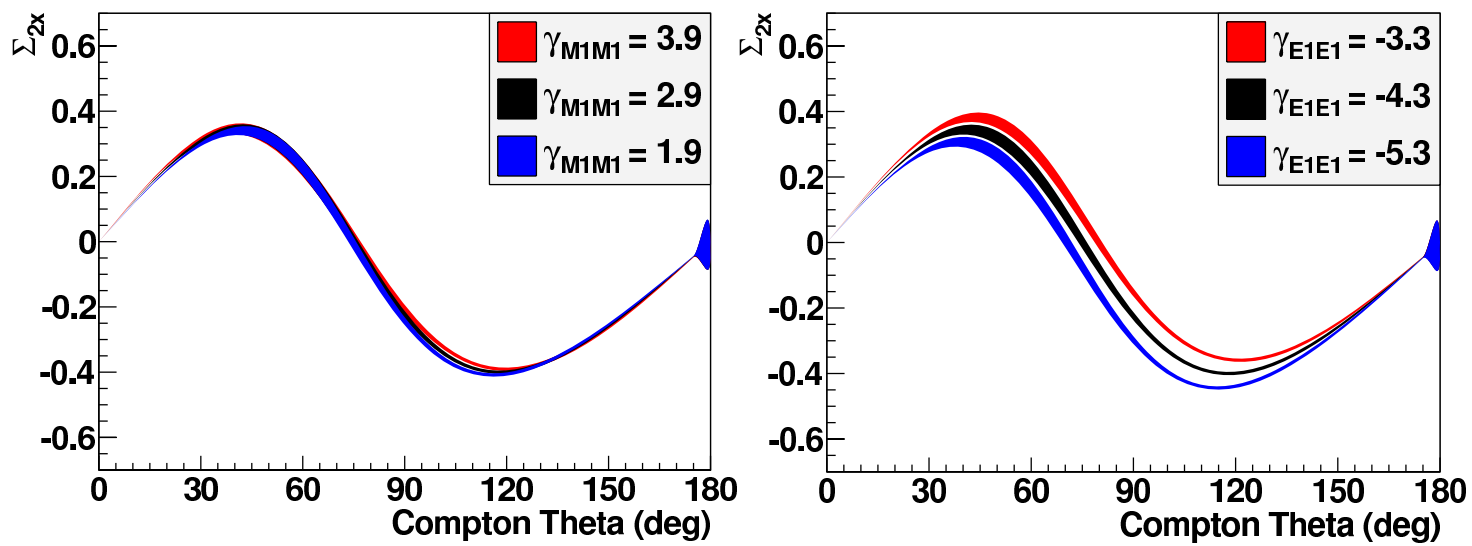

Fig. 1. Left plot, sensitivity to $\gamma_{\mathrm{M} 1 \mathrm{M} 1}$ (see text for details). Right plot, sensitivity to $\gamma_{\mathrm{E} 1 \mathrm{E} 1}$. The incident photon energy is $290 \mathrm{MeV}$.

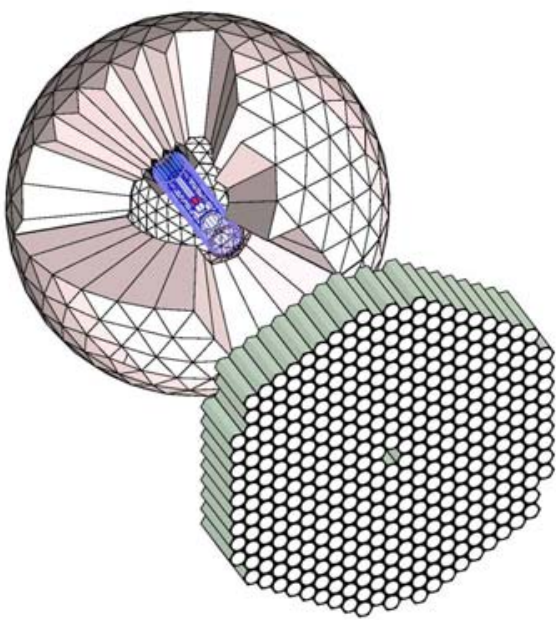

Fig. 2. Diagram of the Crystal Ball and TAPS detectors.

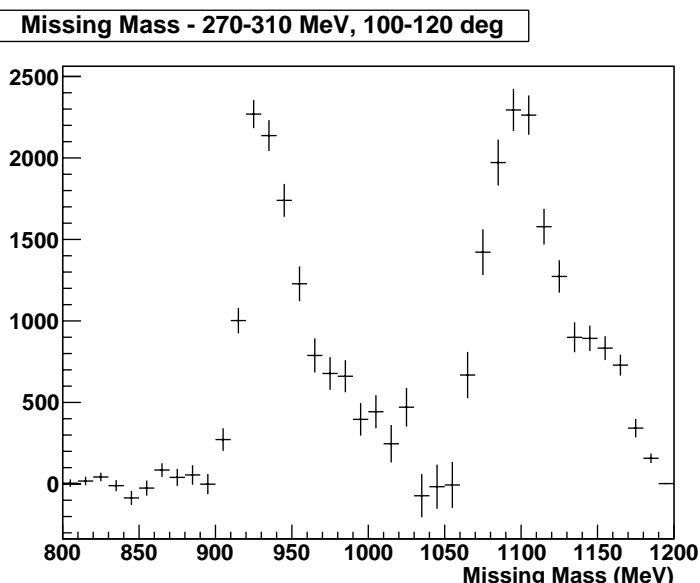

Fig. 3. Missing mass spectrum over incident energies 270 to $310 \mathrm{MeV}$, and scattering angles $100^{\circ}$ to $120^{\circ}$. The vertical axis is counts, the horizontal $\mathrm{MeV}$. 


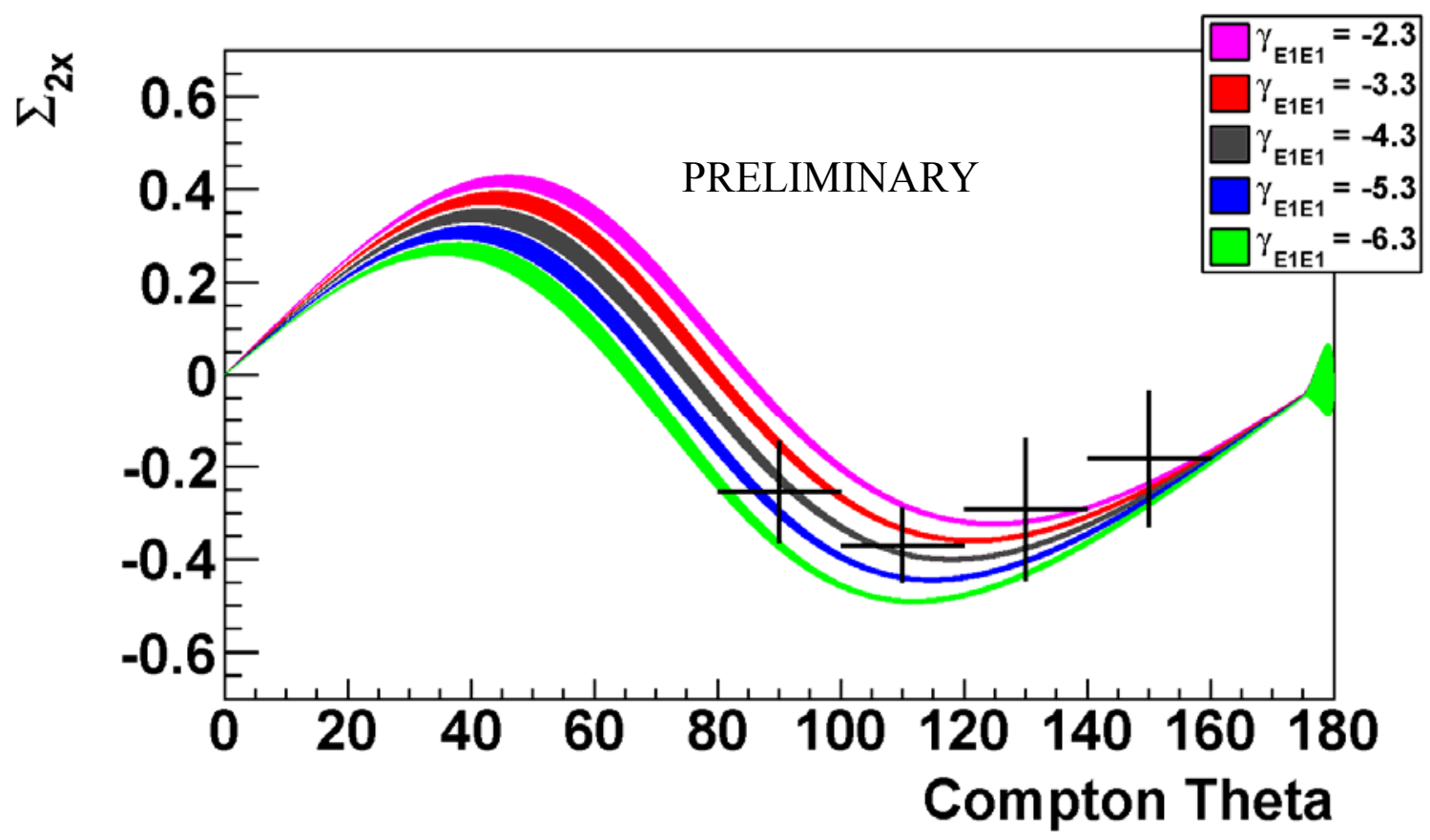

Fig. 4. Preliminary experimental asymmetries. The bands are calculations for different values of $\gamma_{\mathrm{EIEI}}$. 